\title{
Asymptotic properties of stochastic Cahn-Hilliard equation with singular nonlinearity and degenerate noise
}

\author{
Ludovic Goudenège, Luigi Manca ${ }^{\dagger}$
}

\begin{abstract}
We consider a stochastic partial differential equation with a logarithmic nonlinearity with singularities at 1 and -1 and a constraint of conservation of the space average. The equation, driven by a trace-class space-time noise, contains a bi-Laplacian in the drift. We obtain existence of solution for equation with polynomial approximation of the nonlinearity. Tightness of this approximated sequence of solutions is proved, leading to a limit transition semi-group. We study the asymptotic properties of this semi-group, showing the existence and uniqueness of invariant measure, asymptotic strong Feller property and topological irreducibility.
\end{abstract}

\section{Introduction and main results}

The Cahn-Hilliard-Cook equation is a model to describe phase separation in a binary alloy (see [6], [7] and [8]) in the presence of thermal fluctuations (see [11] and [25]). It takes the form

$$
\begin{cases}\partial_{t} u=-\frac{1}{2} \Delta(\Delta u-\psi(u))+\dot{\xi}, & \text { on } \Omega \subset \mathbb{R}^{n}, \\ \nabla u \cdot \nu=0=\nabla(\Delta u-\psi(u)) \cdot \nu, & \text { on } \partial \Omega,\end{cases}
$$

where $t$ denotes the time variable and $\Delta$ is the Laplace operator. Also $u \in[-1,1]$ represents the ratio between the two species and the noise term $\dot{\xi}$ accounts for the thermal fluctuations. The nonlinear term $\psi$ has the double-logarithmic form

$$
\psi: u \mapsto \frac{\theta}{2} \ln \left(\frac{1+u}{1-u}\right)-\theta_{c} u
$$

where $\theta$ and $\theta_{c}$ are temperatures with $\theta<\theta_{c}$.

The unknown $u$ represents the concentration of one specie with respect to the second one. In the deterministic case, the equation is obtained as a gradient in $\mathrm{H}^{-1}(\Omega)$ of the free energy

$$
\mathcal{E}(u)=\int_{\Omega}\left(\frac{1}{2}|\nabla u|^{2}+\Psi(u)\right) \mathrm{d} x
$$

where $\Psi$ is an antiderivative of $\psi$. Since the gradient is taken in $\mathrm{H}^{-1}(\Omega)$, a fourth order equation is obtained. A key point is that the average $\int_{\Omega} u \mathrm{~d} x$ is conserved.

The deterministic equation where $\psi$ is replaced by a polynomial function has first been studied (see [7], [25] and [29]). The drawback of a polynomial nonlinearity is that the solution is not constrained to remain in the physically relevant interval $[-1,1]$. Singular nonlinear terms, such as the logarithmic nonlinearity considered here, remedy this problem. Such non smooth functions $\psi$ have also been considered (see [5] and [15]).

AMS 2000 subject classifications. 60H15, 60H $07,37 \mathrm{~L} 40$

Key words and phrases : ergodicity, Cahn-Hilliard, stochastic partial differential equations, singular nonlinearity, degenerate noise.

*CNRS, LAMA, 77454 Marne-la-Vallée, France, goudenege@math.cnrs.fr

†LAMA, 77454 Marne-la-Vallée, France, luigi.manca@u-pem.fr 
Phase separation has been analyzed thanks to this model: see for example the survey [28], and the references therein, or others recent results on spinodal decomposition and nucleation in $[1,4,22,26,27,32,33,34]$.

The noise term accounts for thermal fluctuations and is a commonly accepted model now. This stochastic equation has also been first studied in the case of a polynomial nonlinearity (see $[2,3,9,10,12,18])$. Again, with a polynomial nonlinear term the solutions do not remain in $[-1,1]$ in general. It is even worse in the presence of noise since any solution leaves this interval immediately with positive probability.

Since the Cahn-Hilliard equation is a gradient flow in $\mathrm{H}^{-1}(\Omega)$, it is natural to consider a noise which is a cylindrical Wiener process in this space (i.e. the spatial derivative of a space-time white noise). Another justification of this choice for the noise is that the stochastic equation has still a gradient structure and an explicit invariant measure is known.

Unfortunately, if the dimension $n$ is larger than 1, it is not difficult to see that with such a noise, even for the linear equation, the solutions have negative spatial regularity and it does not seem possible to treat the nonlinearity. Finally, it is not expected that the singularity of logarithmic nonlinear term is strong enough to prevent the solution from exiting the interval $[-1,1]$. It has been rigorously proved in [21] that a nonlinear term of the form $\ln u$ is not strong enough to ensure that solutions remain positive and a reflection measure has to be added.

The stochastic heat equation with reflection, i.e. when the fourth order operator is replaced by the Laplace operator, is a model for the evolution of random interfaces near a hard wall. It has been extensively studied in the literature (see [14], [19], [20], [30] [35], [36] and [37]). The two-walls case has been treated in [31]. Essential tools in these articles are the comparison principle and the fact that the underlying Dirichlet form is symmetric so that the invariant measure is known explicitly.

However, no comparison principle holds for fourth order equations and new techniques have to be developed. Equation (0.1) has been studied in [17] with a single reflection and when no nonlinear term is taken into account. The reflection is introduced to enforce positivity of the solution. It has been shown in [38] that a conservative random interface model close to one hard wall gives rise to this fourth-order stochastic partial differential equation with one reflection. Various techniques have been introduced to overcome the lack of comparison principle. Moreover, as in the second order case, an integration by part formula for the invariant measure has been derived. Then, in [21], a singular nonlinearity of the form $u^{-\alpha}$ or $\ln u$ has been considered. Existence and uniqueness of solutions have been obtained and using the integration by parts formula as in [36], it has been proved that the reflection measure vanishes if and only if $\alpha \geq 3$. In particular, as mentioned above, for a logarithmic nonlinearity, the reflection is active. For a double singular nonlinearity of the form $(0.2)$, thanks to a delicate a priori estimate on the $\mathrm{L}^{1}$ norm of the nonlinearity, the authors have obtained uniqueness and existence.

In this article, we do not consider that the noise is a cylindrical Wiener process in $\mathrm{H}^{-1}$. In this case the equation has not the desired gradient structure and the technics developed in [16], [17] and [21] are not any more valid. However the choice of smooth-enough space-time noise permits to work with an Itô's formula. Following the approach of [16], we shall consider the problem with a polynomial approximation of the nonlinearity $\psi$. We obtain a priori estimates on the approximated solutions which are sufficient to prove tightness.

Unfortunately, due to the degenerate noise, we are not able to show that the approximated solutions are tight in $\mathcal{C}([0, T] \times[0,1] ;[-1,1])$ and, as consequence, we are not able to characterise the limit process $X(t, x)$ as a solution of some stochastic partial differential equation.

Despite of this, we discuss about the asymptotic properties of the transition semi-group $P_{t}$ associated to this process. The main result of the paper consist in Theorems (3.1) and (3.2), where we show that $P_{t}$ is ergodic and admits a unique invariant measure. The ergodicity result is obtained by assuming that the noise acts on the space spanned by the first eigenvectors of the laplacian (see (3.2), (3.3)) ; this property is sometimes called essentially elliptic (see [23], [24]).

The paper is organised as follows : in the next section we introduce notations and the approximated problem ; in section 2 we obtain some a priori estimate and the convergence of the approximated process to the limit process as well as the associated transition semigroup ; in section 3 we discuss about the ergodicity properties of the transition semigroup. 


\section{Preliminaries}

We denote by $\langle\cdot, \cdot\rangle$ the scalar product in $\mathrm{L}^{2}(0,1) ; A$ is the realization in $\mathrm{L}^{2}(0,1)$ of the Laplace operator with Neumann boundary condition, i.e.

$$
D(A)=\text { Domain of } A=\left\{h \in W^{2,2}(0,1): h^{\prime}(0)=h^{\prime}(1)=0\right\}
$$

where $W^{2,2}(0,1)$ is the classical Sobolev space. Remark that $A$ is self-adjoint on $\mathrm{L}^{2}(0,1)$ and we have a complete orthonormal system of eigenvectors $\left(e_{i}\right)_{i \in \mathbb{N}}$ in $\mathrm{L}^{2}(0,1)$ for the eigenvalues $\left(\lambda_{i}\right)_{i \in \mathbb{N}}$. We denote by $\bar{h}$ the mean of $h \in \mathrm{L}^{2}(0,1)$

$$
\bar{h}=\int_{0}^{1} h(\theta) \mathrm{d} \theta .
$$

We remark that $A$ is invertible on the space of functions with 0 average. In general, we define $(-A)^{-1} h=(-A)^{-1}(h-\bar{h})+\bar{h}$.

For $\gamma \in \mathbb{R}$, we define $(-A)^{\gamma}$ by classical interpolation. We set $V_{\gamma}:=D\left((-A)^{\gamma / 2}\right)$. It is endowed with the classical seminorm and norm

$$
|h|_{\gamma}=\left(\sum_{i=1}^{+\infty}\left(-\lambda_{i}\right)^{\gamma} h_{i}^{2}\right)^{1 / 2},\|h\|_{\gamma}=\left(|h|_{\gamma}^{2}+\bar{h}^{2}\right)^{1 / 2}, \text { for } h=\sum_{i \in \mathbb{N}} h_{i} e_{i} .
$$

$|\cdot|_{\gamma}$ is associated to the scalar product $(\cdot, \cdot)_{\gamma}$. To lighten notations, we set $(\cdot, \cdot):=(\cdot, \cdot)_{-1}$ and $\mathrm{H}:=V_{-1}$. The average can be defined in any $V_{\gamma}$ by $\bar{h}=\left(h, e_{0}\right)$. It plays an important role and we often work with functions with a fixed average $c \in \mathbb{R}$. We define $\mathrm{H}_{c}=\{h \in \mathrm{H}: \bar{h}=c\}$ for all $c \in \mathbb{R}$.

We denote by $\mathcal{B}_{b}\left(\mathrm{H}_{c}\right)$ the space of all Borel bounded functions

The covariance operator of the noise is an operator $B$ such that

$$
\operatorname{Tr}_{\gamma}:=\operatorname{Tr}\left[\sqrt{B}(-A)^{\gamma} \sqrt{B}^{*}\right]
$$

is finite for some $\gamma \in \mathbb{R}$ which will be precise after. Moreover we assume $\sqrt{B} e_{0}=0$ in order to ensure the conservation of average.

For $\lambda \in \mathbb{R}$, we define:

$$
f(u):=\left\{\begin{array}{lr}
+\infty, & \text { for all } u \leq-1, \\
\ln \left(\frac{1-u}{1+u}\right)+\lambda u, & \text { for all } u \in(-1,1), \\
-\infty, & \text { for all } u \geq 1,
\end{array}\right.
$$

and the following antiderivative $F$ of $-f$ :

$$
F(u)=(1+u) \ln (1+u)+(1-u) \ln (1-u)-\frac{\lambda}{2} u^{2}, \text { for all } u \in(-1,1) .
$$

Let $X(\cdot, \cdot)$ stand for a function on $[0, T] \times[0,1]$. With these notations, we rewrite $(0.1)$ in the abstract form:

$$
\left\{\begin{array}{l}
\mathrm{d} X=-\frac{1}{2} A\left(A X+f(X)+\eta_{-}-\eta_{+}\right) \mathrm{d} t+\sqrt{B} \mathrm{~d} W, \\
\left\langle(1+X), \eta_{-}\right\rangle_{O_{T}}=\left\langle(1-X), \eta_{+}\right\rangle_{O_{T}}=0, \\
X(0, \cdot)=x \text { for } x \in \mathrm{H},
\end{array}\right.
$$

where $W$ is a cylindrical Wiener process on $\mathrm{L}^{2}(0,1)$ and

$$
\langle v, \zeta\rangle_{O_{T}}=\int_{[0, T] \times[0,1]} v \mathrm{~d} \zeta
$$


The equation 1.1 is characterized by the two reflection measures $\eta_{ \pm}$. They act as a force to prevent the solution from leaving the physical domain $[-1,1]$. They appears naturally because the logarithmic nonlinearity is not strong enough. It can also be seen as a Lagrange multiplier for the condition " $X(t, x) \in[-1,1]$ for all $t>0$ ". This interpretation is strongly linked to the contact conditions $\left\langle(1+X), \eta_{-}\right\rangle_{O_{T}}=\left\langle(1-X), \eta_{+}\right\rangle_{O_{T}}=0$. In the white noise case studied in [16], the stationary solution has enough regularity to obtain a $\mathrm{L}^{1}\left(O_{T}\right)$ estimate on $f(X)$. This estimates permits to give a sense to $\int_{0}^{T} \int_{0}^{1} f(X(t, \theta)) A h(\theta) \mathrm{d} t \mathrm{~d} \theta$ since $A h \in \mathrm{L}^{\infty}\left(O_{T}\right)$. Uniqueness can be proved under a nice definition of the weak solution as in [16].

The solution of the linear equation with initial data $x \in \mathrm{H}$ is given by

$$
Z(t, \cdot, x)=e^{-t A^{2} / 2} x+\int_{0}^{t} e^{-(t-s) A^{2} / 2} \sqrt{B} \mathrm{~d} W_{s} .
$$

As easily seen this process is in $\mathcal{C}([0,+\infty[; H)$ (see [13]). In particular, the mean of $Z$ is constant and the law of the process $Z$ is the Gaussian measure:

$$
Z(t, \cdot, x) \sim \mathcal{N}\left(e^{-t A^{2} / 2} x, Q_{t}\right), Q_{t}=\int_{0}^{t} \sqrt{B}^{*} e^{-s A^{2} / 2} e^{-s A^{2} / 2} \sqrt{B} \mathrm{~d} s=\sqrt{B}^{*}(-A)^{-1}\left(I-e^{-t A^{2}}\right) \sqrt{B}
$$

If we let $t \rightarrow+\infty$, the law of $Z(t, \cdot, x)$ converges to the Gaussian measure on $\mathrm{L}^{2}$ :

$$
\mu_{c}:=\mathcal{N}\left(c e_{0}, \sqrt{B}(-A)^{-1} \sqrt{B}^{*}\right) \text {, where } c=\bar{x} .
$$

In order to solve equation (1.1), we use polynomial approximations of this equation. We denote by $\left\{f_{n}\right\}_{n \in \mathbb{N}}$ the sequence of polynomial functions which converges to the function $f$ on $(-1,1)$, defined for $n \in \mathbb{N}$ by:

$$
f_{n}(u)=-2 \sum_{k=0}^{n} \frac{u^{2 k+1}}{(2 k+1)}+\lambda u, \text { for all } u \in \mathbb{R} .
$$

We also use in a crucial way that $u \mapsto f_{n}(u)-\lambda u$ is monotone non-increasing below.

Then for $n \in \mathbb{N}$, we study for the following polynomial approximation of (1.1) with an initial condition $x \in \mathrm{H}$ :

$$
\left\{\begin{array}{l}
\mathrm{d} X^{n}+\frac{1}{2}\left(A^{2} X^{n}+A f_{n}\left(X^{n}\right)\right) \mathrm{d} t=\sqrt{B} \mathrm{~d} W \\
X^{n}(0, \cdot)=x
\end{array}\right.
$$

This equation has been studied in [12] in the case $B=I$. The results generalize immediately and it can be proved that for any $x \in \mathrm{H}$, there exists a unique solution $X^{n}(\cdot, \cdot)$ a.s. in $\mathcal{C}([0, T] ; \mathrm{H}) \cap$ $\mathrm{L}^{2 n+2}((0, T) \times(0,1))$. It is a solution in the mild or weak sense. Moreover the average of $X^{n}(t, \cdot)$ does not depend on $t$.

For each $c \in \mathbb{R},(1.2)$ defines a transition semigroup $\left(P_{t}^{n, c}\right)_{t \geq 0}$ :

$$
P_{t}^{n, c} \phi(x)=\mathbb{E}\left[\phi\left(X^{n}(t, \cdot)\right)\right], t \geq 0, x \in \mathrm{H}_{c}, \phi \in \mathcal{B}_{b}\left(\mathrm{H}_{c}\right), n \in \mathbb{N} .
$$

Existence of an invariant measure can be proved as in [12].

In all the article, $\mathrm{C}$ denotes a constant which may depend on parameters and its value may change from one line to another.

\section{A priori estimates in Hilbert spaces and tightness}

In this section we prove the tightness of the solutions of approximated equations, and obtain a limit transition semi-group. Fix $-1<c<1$ and $x$ an initial data in $\mathrm{H}_{c}$. Now consider the unique solution of (1.2) denoted $X^{n}$ in $\mathrm{H}_{c}$ with initial data $x$. We are going to prove that the laws of $\left(X^{n}\right)_{n \in \mathbb{N}}$ are tight in some suitable space. First, we prove a result which only needs the assumption $\operatorname{Tr}_{-1}<+\infty$. 
Proposition 2.1. Suppose $\operatorname{Tr}_{-1}<+\infty$. The laws of $\left(X^{n}\right)_{n \in \mathbb{N}}$ are tight in $\mathrm{L}^{\infty}\left([0, T] ; V_{-1}\right) \cap$ $\mathrm{L}^{2}\left([0, T] ; V_{1}\right)$, and we have

$$
\mathbb{E}\left[\int_{0}^{T}\left|X^{n}(t)\right|_{1}^{2} \mathrm{~d} t\right] \leq|x|_{-1}^{2}+T \mathcal{Q}_{c}(\lambda),
$$

where $\mathcal{Q}_{c}(\lambda)$ is a polynomial.

Proof : Applying Itô formula to $\left|X^{n}(t)\right|_{-1}^{2}$, we obtain

$$
\begin{array}{r}
\left|X^{n}(T)\right|_{-1}^{2}-\left|X^{n}(0)\right|_{-1}^{2}+\int_{0}^{T}\left|X^{n}(t)\right|_{1}^{2} \mathrm{~d} t+2 \int_{O_{T}} \sum_{k=0}^{n} \frac{\left(X^{n}\right)^{2 k+2}}{2 k+1} \mathrm{~d} s \mathrm{~d} \theta+c \int_{O_{T}} \lambda X^{n} \mathrm{~d} s \mathrm{~d} \theta \\
=2 \int_{0}^{T}\left(X^{n}(t), \sqrt{B} \mathrm{~d} W_{t}\right)+T \operatorname{Tr}_{-1}+\int_{O_{T}} \lambda\left(X^{n}\right)^{2} \mathrm{~d} s \mathrm{~d} \theta+2 c \int_{O_{T}} \sum_{k=0}^{n} \frac{\left(X^{n}\right)^{2 k+1}}{(2 k+1)} \mathrm{d} s \mathrm{~d} \theta
\end{array}
$$

Using Hölder's inequality, we have

$$
\begin{aligned}
\sum_{k=0}^{n} c \int_{0}^{1} \frac{\left(X^{n}(t)\right)^{2 k+1}}{2 k+1} \mathrm{~d} \theta & \leq \sum_{k=0}^{n} \frac{c}{2 k+1}\left(\int_{0}^{1}\left(X^{n}(t)\right)^{2 k+2} \mathrm{~d} \theta\right)^{2 k+1 / 2 k+2} \\
& \leq \sum_{k=0}^{n}\left(\frac{1}{2 k+2} \int_{0}^{1}\left(X^{n}(t)\right)^{2 k+2} \mathrm{~d} \theta+\frac{c^{2 k+2}}{(2 k+1)(2 k+2)}\right) .
\end{aligned}
$$

And since

$$
\sum_{k=0}^{n} \int_{0}^{1} \frac{\left(X^{n}(t)\right)^{2 k+2}}{2 k+1} \mathrm{~d} \theta-\sum_{k=0}^{n} \int_{0}^{1} \frac{\left(X^{n}(t)\right)^{2 k+2}}{2 k+2} \mathrm{~d} \theta=\sum_{k=0}^{n} \int_{0}^{1} \frac{\left(X^{n}(t)\right)^{2 k+2}}{(2 k+1)(2 k+2)} \mathrm{d} \theta,
$$

we obtain

$$
\begin{aligned}
\left|X^{n}(T)\right|_{-1}^{2} & -\left|X^{n}(0)\right|_{-1}^{2}+\int_{0}^{T}\left|X^{n}(t)\right|_{1}^{2} \mathrm{~d} t+2 \int_{O_{T}} \sum_{k=0}^{n} \frac{\left(X^{n}(t)\right)^{2 k+2}}{(2 k+1)(2 k+2)} \mathrm{d} t \mathrm{~d} \theta+c \int_{O_{T}} \lambda X^{n}(t) \mathrm{d} t \mathrm{~d} \theta \\
& \leq 2 \int_{0}^{T}\left(X^{n}(t), \sqrt{B} \mathrm{~d} W_{t}\right)+T \operatorname{Tr}_{-1}+\int_{O_{T}} \lambda\left(X^{n}(t)\right)^{2} \mathrm{~d} t \mathrm{~d} \theta+2 T \sum_{k=0}^{n} \frac{c^{2 k+2}}{(2 k+1)(2 k+2)} .
\end{aligned}
$$

Using $\overline{X^{n}(t)}=c$ for all $t \geq 0$, we obtain

$$
\begin{array}{r}
\left|X_{c}^{n}(T)\right|_{-1}^{2}-\left|X^{n}(0)\right|_{-1}^{2}+\int_{0}^{T}\left|X^{n}(t)\right|_{1}^{2} \mathrm{~d} t+c^{2} \lambda T \\
+\int_{O_{T}} \frac{\left(X^{n}(t)\right)^{4}}{6} \mathrm{~d} t \mathrm{~d} \theta+(1-\lambda) \int_{0}^{T}\left|X^{n}(t)\right|_{0}^{2} \mathrm{~d} t+\int_{O_{T}} 2 \sum_{k=2}^{n} \frac{\left(X^{n}(t)\right)^{2 k+2}}{(2 k+1)(2 k+2)} \mathrm{d} t \mathrm{~d} \theta \\
\leq 2 \int_{0}^{T}\left(X^{n}(t), \sqrt{B} \mathrm{~d} W_{t}\right)+T \operatorname{Tr}_{-1}+2 T \sum_{k=0}^{n} \frac{c^{2 k+2}}{(2 k+1)(2 k+2)} .
\end{array}
$$

Remark that

$$
\frac{y^{4}}{6}+(1-\lambda) y^{2}+\frac{3}{2}(1-\lambda)^{2} \geq 0
$$

for any real number $y$. Let $y=X^{n}(t)$ and integrate on $O_{T}$, it follows that

$$
\int_{O_{T}} \frac{\left(X^{n}(t)\right)^{4}}{6} \mathrm{~d} t \mathrm{~d} \theta+(1-\lambda) \int_{0}^{T}\left|X^{n}(t)\right|_{0}^{2} \mathrm{~d} t+\frac{3}{2} T(1-\lambda)^{2} \geq 0 .
$$


Combining (2.2) and (2.3), we obtain

$$
\begin{array}{r}
\left|X c^{n}(T)\right|_{-1}^{2}-\left|X^{n}(0)\right|_{-1}^{2}+\int_{0}^{T}\left|X^{n}(t)\right|_{1}^{2} \mathrm{~d} t+\int_{O_{T}} 2 \sum_{k=2}^{n} \frac{\left(X^{n}(t)\right)^{2 k+2}}{(2 k+1)(2 k+2)} \mathrm{d} t \mathrm{~d} \theta \\
\leq 2 \int_{0}^{T}\left(X^{n}(t), \sqrt{B} \mathrm{~d} W_{t}\right)+T \operatorname{Tr}_{-1}+\frac{3}{2} T(1-\lambda)^{2}-c^{2} \lambda T+2 T \sum_{k=0}^{n} \frac{c^{2 k+2}}{(2 k+1)(2 k+2)} .
\end{array}
$$

Or simply

$$
\begin{aligned}
\left|X^{n}(T)\right|_{-1}^{2}-|X n(0)|_{-1}^{2}+\int_{0}^{T}\left|X^{n}(t)\right|_{1}^{2} \mathrm{~d} t & \leq 2 \int_{0}^{T}\left(X^{n}(t), \sqrt{B} \mathrm{~d} W_{t}\right) \\
& +T\left(\operatorname{Tr}_{-1}+\frac{3}{2}(1-\lambda)^{2}-c^{2} \lambda+F(c)\right)
\end{aligned}
$$

Remark that for all $c \in(-1,1)$ the polynomial function $\mathcal{P}_{c}: \lambda \mapsto \frac{3}{2}(1-\lambda)^{2}-c^{2} \lambda+F(c)$ is always nonnegative. Indeed, if we compute its classical discriminant, we find

$$
\begin{aligned}
\Delta_{\mathcal{P}_{c}} & =\left(c^{2}+3\right)^{2}-6\left(\frac{3}{2}+F(c)\right) \\
& =c^{4}+6 c^{2}+9-9-12 \sum_{k=0}^{+\infty} \frac{c^{2 k+2}}{(2 k+1)(2 k+2)} \\
& =-12 \sum_{k=2}^{+\infty} \frac{c^{2 k+2}}{(2 k+1)(2 k+2)} \leq 0 .
\end{aligned}
$$

So the minimum of $\mathcal{P}_{c}$ is attained in $\lambda^{*}=\frac{c^{2}}{3}+1$ such that

$$
\mathcal{P}_{c}\left(\lambda^{*}\right)=2 \sum_{k=2}^{+\infty} \frac{c^{2 k+2}}{(2 k+1)(2 k+2)} \geq 0
$$

Denote $\mathcal{Q}_{c}(\lambda)=\operatorname{Tr}_{-1}+\mathcal{P}_{c}(\lambda)$. Using Poincaré's inequality and taking the expectation, we obtain

$$
\mathbb{E}\left[\left|X^{n}(T)\right|_{-1}^{2}-\left|X c^{n}(0)\right|_{-1}^{2}+\pi^{4} \int_{0}^{T}\left|X^{n}(t)\right|_{-1}^{2} \mathrm{~d} t\right] \leq T \mathcal{Q}_{c}(\lambda) .
$$

And the Gronwall lemma implies for all $t \in[0, T]$

$$
\mathbb{E}\left[\left|X^{n}(t)\right|_{-1}^{2}\right] \leq\left(\mathbb{E}\left[\left|X^{n}(0)\right|_{-1}^{2}\right]-\frac{\mathcal{Q}_{c}(\lambda)}{\pi^{4}}\right) \exp \left(-\pi^{4} t\right)+\frac{\mathcal{Q}_{c}(\lambda)}{\pi^{4}} .
$$

Under our assumption on the trace class property of the operator $\sqrt{B}$, there there exists a constant $C$ such that

$$
\begin{aligned}
\mathbb{E}\left[\left(\int_{0}^{T}\left(X^{n}(t), \sqrt{B} \mathrm{~d} W_{t}\right)\right)^{2}\right] & =\mathbb{E}\left[\int_{0}^{T}\left|\sqrt{Q B}^{*} X^{n}(t)\right|_{-1}^{2} \mathrm{~d} t\right] \\
& \leq C \mathbb{E}\left[\int_{0}^{T}\left|X^{n}(t)\right|_{-1}^{2} \mathrm{~d} t\right]
\end{aligned}
$$

We set

$$
\begin{aligned}
\varphi_{n}= & \left|X^{n}(T)\right|_{-1}^{2}-\left|X^{n}(0)\right|_{-1}^{2}+\int_{0}^{T}\left|X^{n}(t)\right|_{1}^{2} \mathrm{~d} t-T \mathcal{Q}_{c}(\lambda) \\
& -\int_{O_{T}} f_{n_{k}}\left(X^{n}(t)\right)\left(X^{n}(t)-c\right) \mathrm{d} t \mathrm{~d} \theta
\end{aligned}
$$


such that

$$
\begin{aligned}
M^{2} \mathbb{P}\left(\left|\varphi_{n}\right| \geq M\right) & \leq \mathbb{E}\left[\phi_{n}^{2}\right] \leq \mathbb{E}\left[\left(2 \int_{0}^{T}\left(X^{n}(t), \sqrt{B} \mathrm{~d} W_{t}\right)\right)^{2}\right] \\
& \leq C \mathbb{E}\left[\int_{0}^{T}\left|X_{c}^{n}(t)\right|_{-1}^{2} \mathrm{~d} t\right] \\
& \leq C\left(\left(\mathbb{E}\left[\left|X_{c}^{n}(0)\right|_{-1}^{2}\right]-\frac{\mathcal{Q}_{c}(\lambda)}{\pi^{4}}\right)\left(1-\exp \left(-\pi^{4} T\right)\right)+T \mathcal{Q}_{c}(\lambda)\right) .
\end{aligned}
$$

we obtain

$$
\left|X^{n}(T)\right|_{-1}^{2}-\left|X^{n}(0)\right|_{-1}^{2}+\int_{0}^{T}\left|X^{n}(t)\right|_{1}^{2} \mathrm{~d} t \leq 2 \int_{0}^{T}\left(X^{n}(t), \sqrt{B} \mathrm{~d} W_{t}\right)+T \mathcal{Q}_{c}(\lambda),
$$

giving the boundedness of expectation in $\mathrm{L}^{2}\left([0, T] ; V_{1}\right)$

$$
\mathbb{E}\left[\int_{0}^{T}\left|X^{n}(t)\right|_{1}^{2} \mathrm{~d} t\right] \leq \mathbb{E}\left[\left|X^{n}(0)\right|_{-1}^{2}\right]+T \mathcal{Q}_{c}(\lambda) .
$$

Using Poincaré's inequality, and taking the expectation, we obtain

$$
\mathbb{E}\left[\left|X^{n}(T)\right|_{-1}^{2}\right]+\pi^{4} \mathbb{E}\left[\int_{0}^{T}\left|X^{n}(t)\right|_{-1}^{2} \mathrm{~d} t\right] \leq \mathbb{E}\left[\left|X^{n}(0)\right|_{-1}^{2}\right]+T \mathcal{Q}_{c}(\lambda) .
$$

Using Gronwall lemma implies for all $t \in[0, T]$

$$
\mathbb{E}\left[\left|X^{n}(t)\right|_{-1}^{2}\right] \leq\left(\mathbb{E}\left[\left|X^{n}(0)\right|_{-1}^{2}\right]-\frac{\mathcal{Q}_{c}(\lambda)}{\pi^{4}}\right) \exp \left(-\pi^{4} t\right)+\frac{\mathcal{Q}_{c}(\lambda)}{\pi^{4}},
$$

giving the desired boundedness of expectation in $\mathrm{L}^{\infty}\left([0, T] ; V_{-1}\right) \cap \mathrm{L}^{2}\left([0, T] ; V_{1}\right)$.

Proposition 2.2. Suppose $x \in V_{0}$ and $\operatorname{Tr}_{0}<+\infty$. Then the laws of $\left(X^{n}\right)_{n \in \mathbb{N}}$ are tight in $\mathrm{L}^{2}\left([0, T] ; V_{2}\right) \cap \mathrm{L}^{\infty}\left([0, T] ; V_{0}\right)$, and we have

$$
2 \int_{O_{T}}\left(\nabla X^{n}(t, \theta)\right)^{2} \sum_{k=0}^{n}\left(X^{n}(t, \theta)\right)^{2 k} \mathrm{~d} t \mathrm{~d} \theta \leq 2 \int_{0}^{T}\left\langle\sqrt{B}^{*} X^{n}(t), \mathrm{d} W_{t}\right\rangle+|x|_{0}^{2}+T \operatorname{Tr}_{0} .
$$

Proof : Applying Ito formula to $\left|X^{n}(t)\right|_{0}^{2}$, we obtain

$$
\begin{aligned}
\left|X^{n}(T)\right|_{0}^{2}-\left|X^{n}(0)\right|_{0}^{2} & +\int_{0}^{T}\left|X^{n}(t)\right|_{2}^{2} \mathrm{~d} t+2 \int_{O_{T}} \nabla X^{n}(t) \sum_{k=0}^{n} \frac{\nabla\left(\left(X^{n}(t)\right)^{2 k+1}\right)}{2 k+1} \mathrm{~d} t \mathrm{~d} \theta \\
& =-\lambda \int_{O_{T}}\left(\nabla X^{n}\right)^{2} \mathrm{~d} s \mathrm{~d} \theta+2 \int_{0}^{T}\left\langle X^{n}(t), \sqrt{B} \mathrm{~d} W_{t}\right\rangle+T \operatorname{Tr}_{0} \\
=\left|X^{n}(T)\right|_{0}^{2}-\left|X^{n}(0)\right|_{0}^{2} & +\int_{0}^{T}\left|X^{n}(t)\right|_{2}^{2} \mathrm{~d} t+4 \int_{O_{T}}\left(\nabla X^{n}(t)\right)^{2}\left(\sum_{k=0}^{n}\left(X^{n}(t)\right)^{2 k}\right) \mathrm{d} t \mathrm{~d} \theta .
\end{aligned}
$$

Eliminating the positive terms, we obtain the desired estimation. Moreover taking the expectation, we obtain

$$
\mathbb{E}\left[\int_{0}^{T}\left|X^{n}(t)\right|_{2}^{2} \mathrm{~d} t\right] \leq \mathbb{E}\left[\left|X^{n}(0)\right|_{0}^{2}\right]+T \operatorname{Tr}_{0} .
$$

Using Poincaré's inequality, taking the expectation, and using Gronwall lemma implies for all $t \in[0, T]$

$$
\mathbb{E}\left[\left|X^{n}(t)\right|_{0}^{2}\right] \leq\left(\mathbb{E}\left[\left|X^{n}(0)\right|_{0}^{2}\right]-\frac{\operatorname{Tr}_{0}}{\pi^{4}}\right) \exp \left(-\pi^{4} t\right)+\frac{\operatorname{Tr}_{0}}{\pi^{4}}
$$


By Proposition 2.1 and classical diagonalization procedure, we obtain

Proposition 2.3. For all $c \in(-1,1)$, there exists a transition semi-group $P_{t}^{c}, t \geq 0$ on $\mathcal{B}_{c}(\mathrm{H})$ and a subsequence $\left\{n_{k}\right\}$ such that $P_{t}^{n_{k}, c} \varphi(x) \rightarrow P_{t}^{c} \varphi(x)$ as $n_{k} \rightarrow \infty$, for all $\varphi \in \mathcal{B}_{c}(\mathrm{H}), x \in \mathrm{H}, t \geq 0$.

\section{Ergodicity properties}

Existence of an invariant measure follows by a classical argument of compactness and by the estimate

$$
\left|X^{n}(t, x)-X^{n}(t, y)\right|_{-1} \leq e^{\lambda t}\|x-y\|_{-1}
$$

which holds any $x, y \in \mathrm{H}_{c}, t>0$.

Theorem 3.1. For any $c \in(-1,1)$ there exists an invariant measure for the semigroup $P_{t}^{c}, t \geq 0$.

Proof. Fix $c \in(-1,1)$. By $(2.1)$ and by the Krylov-Bogoliubov criterion, for any $n \in \mathbb{N}$ there exists an invariant measure for the transition semigroup $P^{c, n}, t \geq 0$. Let $\nu$ be as a weak ${ }^{*}$ limit of the sequence $\left\{\nu^{n}\right\}$. By using (3.1) and the thightness of $\nu^{n}$ it is strightforward to verify that $\nu$ is invariant for $P_{t}$.

In order to show uniqueness of such invariant measure, we shall show (under some suitable assumption on the noise, see (3.2)) that its transition semigroup $P_{t}^{c}$ enjoys the asymptotic strong Feller property and it is topologically irreducible. We recall that since the noise in degenerate, we are not able to use the same techniques of [21]; in particular, the Bismut-Elworthy formula does not apply, and we are not able to show that the semigroup $P_{t}^{c}$ is strong Feller.

Asymptotic strong Feller property was introduced in [23] in order to study ergodicity properties of a $2 D$ Navier-Stokes equation perturbed by a very degenerate noise. The exact definition can be found in [23, Definition 3.8]. Here we consider the property in the following form, which shall be sufficient for our pourpose:

Proposition 3.1 (Proposition 3.12 in [23]). Let $t_{n}$ and $\delta_{n}$ be two positive sequences with $\left\{t_{n}\right\}$ nondecreasing and $\left\{\delta_{n}\right\}$ converging to zero. A semigroup $P_{t}$ on a Hilbert space $H$ is asymptotically strong Feller if, for all $\varphi: H \rightarrow \mathbb{R}$ with $|\varphi|_{\infty}$ and $|\nabla \varphi|_{\infty}$ finite,

$$
\left|\nabla P_{t} \varphi(x)\right| \leq C\left(\|x\|_{H}\right)\left(|\varphi|_{\infty}+\delta_{n}|\nabla \varphi|_{\infty}\right)
$$

for all $n$, where $C: \mathbb{R}^{+} \rightarrow \mathbb{R}^{+}$is a fixed nondecrasing function.

Unfortunately, we are not able to show that the semigroup $P_{t}^{c}$ is differentiable (in the case of a white noise perturbation the semigroup is differentiable, and the strong Feller property holds, see [21]). Also for the semigroup $P_{t}^{c, n}$, associated to the approximation (1.2) we are not able to show its differentiability. However, we shall use the following variation of the property

Proposition 3.2. A semigroup $P_{t}$ on a Hilbert space $H$ is asymptotically strong Feller if there exist two constant $C, \delta>0$ such that for all $\varphi: H \rightarrow \mathbb{R}$ with $|\varphi|_{\infty}$ and $|\nabla \varphi|_{\infty}$ finite,

$$
\left|P_{t} \varphi(x)-P_{t} \varphi(y)\right| \leq C\left(|x|_{-1} \vee|y|_{-1}\right)\left(|\varphi|_{\infty}+e^{-\delta t}|\nabla \varphi|_{\infty}\right)|x-y|_{H} .
$$

This proposition can be proved with the same argument used to prove Proposition 3.12 in [23]. The right-hand side is clearly derived by the previous proposition, setting $\delta_{n}=e^{-\delta t}$. The only modification consists in the left-side part, where no assumptions on differentiability of $P_{t} \varphi$ are needed.

The main assumption of this section is

$$
B=\sum_{k=1}^{\infty} b_{k}\left\langle\cdot, e_{k}\right\rangle e_{k}
$$


where $b_{k}>0$ for $k \in\{1, \ldots, N\}$ and

$$
\frac{1}{2}(N+1)^{2}-\lambda>0 .
$$

In other words, we assume that for a sufficiently large $N, \operatorname{span}\left\{e_{0}, \ldots, e_{N}\right\} \subset \operatorname{range}(B)$. This kind of setting is known as essentially elliptic (see [21]). We think that more degenerate noises can be considered in order to have asymptotic strong Feller property, and this shall be the objet of a forthcoming paper.

The importance of the asymptotic strong Feller property is that in this case any two distinct ergodic invariant measures have disjoint topological support (see Theorem 3.16 in [23]). According to [24], we introduce the following

Definition 3.1. We say that a semigroup $P_{t}$ on a Hilbert space $H$ is weakly topologically irreducible if for all $x_{1}, x_{2} \in H$ there exists $y \in H$ so that for any open set $A$ containing $y$ there exist $t_{1}, t_{2}>0$ with $P_{t_{i}}\left(x_{i}, A\right)>0$.

The main result of this section is the following

Theorem 3.2. Under assumptions (3.2) and (3.3), for any $c \in(-1,1)$ the semigroup $P_{t}^{c}, t \geq 0$ has an unique invariant measure.

Proof. By Corollary 1.4 from [24], a Markov semigroup which is Feller, weakly topologically irreducible and asymptotically strong Feller admits at most one invariant probability measure. The proof then follows by Proposition 3.3 and Proposition 3.4.

In order to proceed, we need some apriori estimates on the solution of the approximated problem. Le us denote by $X^{n}(t, x, W)$ the solution of (1.2) perturbed by the Wiener process $W$.

Lemma 3.1. There exist $\delta>0$ and a continuous non decreasing function $C: \mathbb{R}^{+} \rightarrow \mathbb{R}^{+}$such that any $x, y \in \mathrm{H}_{c}, n \in \mathbb{N}$ there exists a process $w_{n} \in L^{2}(0, \infty ; H)$, adapted to $W$, satifying

$$
\left|X^{n}\left(t, x, W+\int_{0}^{.} w_{n}(s) d s\right)-X^{n}(t, y, W)\right|_{-1} \leq e^{-\delta t}|x-y|_{-1}
$$

and

$$
\mathbb{E}\left|1-e^{-\frac{1}{2} \int_{0}^{t} w_{n}^{2}(s) d s+\int_{0}^{t} w_{n}(s) d W(s)}\right| \leq C\left(|x|_{-1} \vee|y|_{-1}\right)|x-y|_{-1} .
$$

Proof. Fix $n \in \mathbb{N}$. Let us denote by $\pi_{l}$ the projection of $\mathrm{H}_{c}$ into $\operatorname{span}\left\{e_{0}, \ldots, e_{N}\right\}$ (the low frequences) and by $\pi_{h}=I-\pi_{l}$ the projection into its orthogonal complement (the high frequences). Let $\tilde{X}$ be the solution of

$$
\left\{\begin{array}{l}
d \tilde{X}=\left(-A^{2} \tilde{X}+\lambda A \pi_{h} \tilde{X}-A p_{n}(\tilde{X})+\lambda A \pi_{l} X^{n}(t, y, W)\right) d t+B d W(t) \\
\tilde{X}(0)=x
\end{array}\right.
$$

Here $p_{n}$ is the increasing polynomial $f_{n}-\lambda$. Arguing as in the previous section, it is strightforward to show that this equation admits a unique solution in $C\left([0, T] ; \mathrm{H}_{c}\right) \cap L^{2}\left([0, T] ; V_{1}\right), T>0$, adapted to $W(t), t \geq 0$. Now set $w_{n}(t)=B^{-1} A \pi_{l}\left(\tilde{X}-X^{n}(t, y, W)\right)$. It is clear that the operator $B^{-1} A \pi_{l}$ : $\mathbb{R}^{N} \rightarrow \mathbb{R}^{N}$ is well defined (thanks to (3.2), (3.3)) and that $w_{n}(t)$ is square integrable and adapted to $W(t), t \geq 0$. Moreover, we find that $X^{n}\left(t, x, W+\int_{0} w_{n}(s) d s\right)$ satisfies the same equation of $\tilde{X}$, so that it has to coincide with $\tilde{X}$.

$$
\begin{aligned}
& \text { Consequently, } Y(t):=X\left(t, x, W+\int_{0} w_{n}(s) d s\right)-X(t, y, W) \text { solves } \\
& \left\{\begin{array}{l}
d Y(t)=\left(-A^{2} Y(t)+\lambda A \pi_{h} Y(t)-A p_{n}\left(X\left(t, x, W+\int_{0} w_{n}(s) d s\right)\right)+A p_{n}(X(t, y, W)) d t\right. \\
Y(0)=x-y .
\end{array}\right.
\end{aligned}
$$


By taking the scalar product of both members with $A^{-1} Y(t)$ and using the dissipativity of $p_{n}$ we find

$$
\begin{aligned}
\frac{1}{2} d|Y(t)|_{-1}^{2}=-|Y(t)|_{1}^{2}+\lambda\left\|\pi_{h} Y(t)\right\|^{2}-\left(p _ { n } \left(X^{n}(t,\right.\right. & \left.\left., W+\int_{0}^{\cdot} w_{n}(s) d s\right)\right)-p_{n}\left(X^{n}(t, y, W), Y(t)\right) \\
\leq & -\left(\frac{1}{2}(N+1)^{2}-\lambda\right)\left|\pi_{h} Y(t)\right|_{0}^{2}-\left|\pi_{l} Y(t)\right|_{0}^{2} .
\end{aligned}
$$

Here we have used the estimate

$$
|\xi|_{1}^{2}=\left|\pi_{l} \xi\right|_{1}^{2}+\left|\pi_{h} \xi\right|_{1}^{2}=\left|\pi_{l} \xi\right|_{1}^{2}+\sum_{k=N+1}^{\infty} k^{2}\left(\xi_{k}\right)^{2} \geq\left|\pi_{l} \xi\right|_{0}^{2}+(N+1)^{2}\left|\pi_{h} \xi\right|_{0}^{2}
$$

where $\xi \in \mathrm{H}_{0}$ and $\xi_{k}$ is the $k$-th Fourier mode. Setting $\delta=2 \pi \min \left\{\frac{1}{2}(N+1)^{2}-\lambda, 1\right\}$ (which is stricly positive by (3.3)) we deduce, by Gronwall lemma,

$$
|Y(t)|_{-1}^{2}+\pi \delta \int_{0}^{t}\|Y(s)\|^{2} d s \leq|x-y|_{-1}^{2} .
$$

Moreover, since $V_{0} \subset V_{-1}$, with $|x|_{-1} \leq \pi^{-1}\|x\|$ we deduce

$$
|Y(t)|_{-1} \leq e^{-\delta t}|x-y|_{-1} .
$$

This proves (3.4). Let us show (3.5). By the definition of $w_{n}$ and by (3.4) we have

$$
\left|w_{n}(s)\right| \leq\left\|B^{-1} A \pi_{l}\right\|_{\mathcal{L}\left(\mathbb{R}^{N} ; \mathbb{R}^{N}\right)}\left|\tilde{X}-X^{n}(s, y, W)\right|_{-1} \leq\left\|B^{-1} A \pi_{l}\right\|_{\mathcal{L}\left(\mathbb{R}^{N} ; \mathbb{R}^{N}\right)} e^{-\delta s}|x-y|_{-1} .
$$

Notice that this estimate holds in $L^{\infty}(\Omega)$. Then the process

$$
e^{-\frac{1}{2} \int_{0}^{t} w_{n}^{2}(s) d s+\int_{0}^{t} w_{n}(s) d W(s)}
$$

is a square integrable martingale and by the well known properties of the exponential martingales it holds

$$
\begin{aligned}
E \mid 1 & -e^{-\frac{1}{2} \int_{0}^{t} w_{n}^{2}(s) d s+\int_{0}^{t} w_{n}(s) d W(s)}|=\mathbb{E}| \int_{0}^{t} e^{-\frac{1}{2} \int_{0}^{s} w_{n}^{2}(\tau) d \tau+\int_{0}^{s} w_{n}(\tau) d W(\tau)} w_{n}(s) d W(s) \mid \\
& \leq\left(\mathbb{E} \int_{0}^{t} e^{-\int_{0}^{s} w_{n}^{2}(\tau) d \tau+2 \int_{0}^{s} w_{n}(\tau) d W(\tau)} w_{n}^{2}(s) d s\right)^{\frac{1}{2}} \\
& \leq\left(\int_{0}^{t} \mathbb{E}\left[e^{-\int_{0}^{s} w_{n}^{2}(\tau) d \tau+2 \int_{0}^{s} w_{n}(\tau) d W(\tau)}\right]\left|w_{n}(s)\right|_{L^{\infty}(\Omega)}^{2} d s\right)^{\frac{1}{2}} \\
& \leq\left(\int_{0}^{t} \mathbb{E}\left[e^{-2 \int_{0}^{s} w_{n}^{2}(\tau) d \tau+2 \int_{0}^{s} w_{n}(\tau) d W(\tau)}\right] e^{\int_{0}^{s}\left|w_{n}(\tau)\right|_{L^{\infty}(\Omega)}^{2} d \tau}\left|w_{n}(s)\right|_{L^{\infty}(\Omega)}^{2} d s\right)^{\frac{1}{2}} \\
& =\left(\int_{0}^{t} e^{\int_{0}^{s}\left|w_{n}(\tau)\right|_{L^{\infty}(\Omega)}^{2} d \tau}\left|w_{n}(s)\right|_{L^{\infty}(\Omega)}^{2} d s\right)^{\frac{1}{2}} \\
& =\left(e^{\int_{0}^{t}\left|w_{n}(\tau)\right|_{L^{\infty}(\Omega)}^{2} d \tau}-1\right)^{\frac{1}{2}} \\
& \leq e^{\frac{1}{2} \int_{0}^{t}\left|w_{n}(\tau)\right|_{L^{\infty}(\Omega)}^{2} d \tau}\left(\int_{0}^{t}\left|w_{n}(\tau)\right|_{L^{\infty}(\Omega)}^{2} d \tau\right)^{\frac{1}{2}} .
\end{aligned}
$$

By (3.6) we have

$$
\begin{aligned}
\int_{0}^{t}\left|w_{n}(\tau)\right|_{L^{\infty}(\Omega)}^{2} d \tau & \leq\left\|B^{-1} A \pi_{l}\right\|_{\mathcal{L}\left(\mathbb{R}^{N} ; \mathbb{R}^{N}\right)}^{2}|x-y|_{-1}^{2} \int_{0}^{t} e^{-2 \delta \tau} d \tau \\
& \leq \frac{\left\|B^{-1} A \pi_{l}\right\|_{\mathcal{L}\left(\mathbb{R}^{N} ; \mathbb{R}^{N}\right)}^{2}|x-y|_{-1}^{2} .}{2 \delta}
\end{aligned}
$$


Then (3.5) is satisfied with

$$
C(t)=\frac{\left\|B^{-1} A \pi_{l}\right\|_{\mathcal{L}\left(\mathbb{R}^{N} ; \mathbb{R}^{N}\right)}}{\sqrt{2 \delta}} \exp \left(\frac{\left\|B^{-1} A \pi_{l}\right\|_{\mathcal{L}\left(\mathbb{R}^{N} ; \mathbb{R}^{N}\right)}^{2}}{2 \delta} t^{2}\right), \quad t \geq 0 .
$$

The previous lemma means that we can find a Wiener process $W^{\prime}$ such that the solution of equation (1.2) starting by $x$ driven by the Wiener process $W^{\prime}$ approches the solution of (1.2) starting by $y$ as $t \rightarrow \infty$. Moreover, this perturbation is uniformly controlled, in the sense of (3.5), by a constant independent by $n$.

Proposition 3.3. Assume that (3.2), (3.3) hold. Then for any $c \in(-1,1)$ the semigroup $P_{t}^{c}$ on $\mathrm{H}_{c}$ has the asymptotic strong Feller property.

Proof : Fix $c \in(-1,1)$. Taking into account Proposition 3.2, it is sufficient to show that there exist a nondecreasing function $C: \mathbb{R}^{+} \rightarrow \mathbb{R}^{+}$and a constant $\delta>0$ such that for any $\psi: \mathrm{H}_{c} \rightarrow \mathbb{R}$ continuous and bounded with bounded Fréchet derivative it holds

$$
\left|P_{t}^{c} \psi(x)-P_{t}^{c} \psi(y)\right| \leq C\left(|x|_{-1} \vee|y|_{-1}\right)\left(|\psi|_{\infty}+e^{-\delta t}|\nabla \psi|_{\infty}\right)|x-y|_{-1} .
$$

Taking into account that $P_{t}^{c, n} \stackrel{n \rightarrow \infty}{\longrightarrow} P_{t}^{c}$ (see Proposition 2.3), for any $\varepsilon>0$ there exists $n \in \mathbb{N}$ (depending on $\varepsilon, \psi, x$ and $y$ ) such that

$$
\left|P_{t}^{c} \psi(x)-P_{t}^{c} \psi(y)\right| \leq \varepsilon+\left|P_{t}^{c, n} \psi(x)-P_{t}^{c, n} \psi(y)\right| .
$$

By the previous Lemma, we take $w_{n}(\cdot)$ such that (3.4), (3.5) hold. Then,

$$
\begin{aligned}
P_{t}^{c, n} \psi(x)-P_{t}^{c, n} \psi(y) & =\mathbb{E} \psi\left(X^{n}(t, x, W)\right)-\mathbb{E} \psi\left(X^{n}(t, y, W)\right)=\mathbb{E} \psi\left(X^{n}(t, x, W)\right) \\
& -\mathbb{E} \psi\left(X^{n}\left(t, x, W+\int_{0} w_{n}(s) d s\right)\right)+\mathbb{E} \psi\left(X^{n}\left(t, y, W+\int_{0} w_{n}(s) d s\right)\right)-\mathbb{E} \psi\left(X^{n}(t, y, W)\right) .
\end{aligned}
$$

We stress that the expectation is taken with respect to the Wiener process $W(t), t \geq 0$. Then, by Girsanov theorem, we obtain

$$
\begin{aligned}
\mathbb{E} \psi\left(X^{n}(t, x, W)\right)-\mathbb{E} \psi\left(X ^ { n } \left(t, x, W+\int_{0}\right.\right. & \left.\left.w_{n}(s) d s\right)\right) \\
& =\mathbb{E}\left[\psi\left(X^{n}(t, x, W)\right)\left(1-e^{-\frac{1}{2} \int_{0}^{t} w_{n}^{2}(s) d s+\int_{0}^{t} w_{n}(s) d W(s)}\right)\right] .
\end{aligned}
$$

Then by (3.5) we deduce

$$
\begin{aligned}
\mid \mathbb{E} \psi\left(X^{n}(t, x, W)\right) & -\mathbb{E} \psi\left(X^{n}\left(t, x, W+\int_{0}^{\cdot} w_{n}(s) d s\right)\right) \mid \\
\leq & |\psi|_{L^{\infty}} \mathbb{E}\left|1-e^{-\frac{1}{2} \int_{0}^{t} w_{n}^{2}(s) d s+\int_{0}^{t} w_{n}(s) d W(s)}\right| \leq C\left(|x|_{-1} \vee|y|_{-1}\right)|\psi|_{L^{\infty}}|x-y|_{-1}
\end{aligned}
$$

On the other side, by (3.4) we obtain

$$
\begin{aligned}
& \left|\mathbb{E} \psi\left(X^{n}\left(t, y, W+\int_{0} w_{n}(s) d s\right)\right)-\mathbb{E} \psi\left(X^{n}(t, y, W)\right)\right| \\
& \quad \leq|\nabla \psi| \mathbb{E}\left|X^{n}\left(t, y, W+\int_{0} w_{n}(s) d s\right)-X^{n}(t, y, W)\right| \leq|\nabla \psi| e^{-\delta t}|x-y|_{-1}
\end{aligned}
$$

Therefore it holds

$$
\left|P_{t}^{c, n} \psi(x)-P_{t}^{c, n} \psi(y)\right| \leq C\left(|x|_{-1} \vee|y|_{-1}\right)|\psi|_{L^{\infty}}|x-y|_{-1}+|\nabla \psi| e^{-\delta t}|x-y|_{-1}
$$

where the continuous nondecreasing function $C: \mathbb{R}^{+} \rightarrow \mathbb{R}^{+}$and the positive constant $\delta$ are independent by $n$ and by $\varepsilon$. Consequently, (3.7) follows. 
Proposition 3.4. For any $c \in(-1,1)$, the semigroup $P_{t}^{c}, t \geq 0$ on $\mathrm{H}_{c}$ is weakly topologically irreducible.

Proof. We shall show that that for any $x \in \mathrm{H}_{c}, \delta>0$ there exists $\varepsilon>0$ and $t>0$ such that $P_{t}^{c}(x, \overline{B(0, \delta)}) \leq 1-\varepsilon$, where $\overline{B(0, \delta)}=\left\{y \in \mathrm{H}_{c}:|y-c|_{-1}>\delta\right\}$. Since $P_{t}^{n, c} \rightarrow P_{t}^{c}$, it is sufficient to show that for such $\left.t>0, P_{t}^{c, n}(x, \overline{B(0, \delta)})^{c}\right) \leq 1-\varepsilon$ with $\varepsilon>0$ independent by $n$. Let $W_{A}(t, x)$ be the solution of

$$
\left\{\begin{array}{l}
D Z=A^{2} Z d t+B d W(t) \\
Z(0)=x
\end{array}\right.
$$

Setting $Y^{n}=X^{n}-W_{A}(t)$, we have that $Y^{n} \in \mathrm{H}_{0}$ and it sastisfies the equation

$$
\left\{\begin{array}{l}
d Y^{n}(t)=A\left(-A Y^{n}+\lambda Y^{n}+f_{n}\left(Y^{n}+W_{A}\right)\right) d t, \\
Y^{n}(0)=0 .
\end{array}\right.
$$

By multiplying both sides by $(-A)^{-1} Y^{n}$ and integrating on $[-1,1]$ we find

$$
\begin{aligned}
\frac{1}{2} d\left|Y^{n}\right|_{-1}^{2} & =-\left\|\nabla Y^{n}\right\|_{2}^{2}+\lambda\left\|Y^{n}\right\|_{2}^{2}+\left\langle f_{n}\left(Y^{n}+W_{A}\right), Y^{n}\right\rangle \\
& \leq-\left\|\nabla Y^{n}\right\|_{2}^{2}+\lambda\left\|Y^{n}\right\|_{2}^{2}+\left\langle f_{n}\left(W_{A}\right), Y^{n}\right\rangle \\
& \leq-\left\|\nabla Y^{n}\right\|_{2}^{2}+\lambda\left\|Y^{n}\right\|_{2}^{2}+\left\|f_{n}\left(W_{A}\right)\right\|_{2}^{2}+4\left\|Y^{n}\right\|_{2}^{2}
\end{aligned}
$$

where we have used Young's inequality and the fact $\left(f_{n}(a+b)-f_{n}(a)\right) b \leq 0$ for any $a, b \in \mathbb{R}$ and $n \geq 0$. It is clear that there exists $C>0$ sucht that

$$
-\|\nabla z\|_{2}^{2}+(\lambda+4)\|z\|_{2}^{2} \leq C|z|_{-1}^{2}
$$

for any $z \in H^{1}$. Then we deduce

$$
\frac{1}{2} d\left|Y^{n}\right|_{-1}^{2} \leq C\left|Y^{n}\right|_{-1}^{2}+\left\|f_{n}\left(W_{A}\right)-f_{n}(c)\right\|_{2}^{2} .
$$

So by Grownwall inequality we find

Consequently,

$$
\left|Y^{n}(t)\right|_{-1}^{2} \leq \int_{0}^{t} e^{2 C(t-s)}\left\|f_{n}\left(W_{A}(s)\right)\right\|_{2}^{2} d s .
$$

$$
\begin{aligned}
\mathbb{P}\left(\left|X^{n}(t)\right|_{-1}>\delta\right) & \leq \mathbb{P}\left(\left|Y^{n}(t)\right|_{-1}+\left|W_{A}(t)\right|_{-1}>\delta\right) \leq \mathbb{P}\left(\left|Y^{n}(t)\right|_{-1}^{2}+\left|W_{A}(t)\right|_{-1}^{2}>\delta^{2} / 2\right) \\
& \leq \mathbb{P}\left(\int_{0}^{t} e^{2 C(t-s)}\left\|f_{n}\left(W_{A}(s)\right)\right\|_{2}^{2} d s+\left|W_{A}(t)\right|_{-1}^{2}>\delta^{2} / 2\right) .
\end{aligned}
$$

By the properties of the approximating functions $f_{n}$ we have $\left|f_{n}\left(W_{A}(s)\right)\right| \leq\left|f\left(W_{A}(s)\right)\right|$ where the right-hand side is allowed to be $+\infty$ if $\left|W_{A}(t, x)\right| \geq 1$. This implies that (3.8) is bounded by

$$
\mathbb{P}\left(\int_{0}^{t} e^{2 C(t-s)}\left\|f\left(W_{A}(s)\right)\right\|_{2}^{2} d s+\left|W_{A}(t)\right|_{-1}^{2}>\delta^{2} / 2\right) .
$$

In order to conclude the proof, it is sufficient to observe that by the gaussianity of the stochastic convolution this probability is $<1$.

\section{Conclusion}

We obtain the ergodicity of the limit semi-group by assuming an essentially elliptic structure of the noise (that is the noise acts on the first modes). We expect that these properties (expecially the ASF property) hold also with more general noises but this need a better understanding of how the nonlinearity spreads the noise.

Unfortunately the structure of the limit equation is unknown. In previous papers, for the same equation perturbed by white noise, it is proved that the nonlinearity has lead to reflection measures. On the other side, in the deterministic case, there is a $L^{\infty}$ bound proving that the solution never approach the singularities. In both cases they prove regularity in space that we have been unable to obtain in our degenerate noise case. With enough regularity, we expect a good characterization of the solution (with or without reflection measure) leading to uniqueness. 


\section{References}

[1] P. W. Bates and P. C. Fife. The dynamics of nucleation for the Cahn-Hilliard equation. SIAM J. Appl. Math., 53(4):990-1008, 1993.

[2] D. Blömker, S. Maier-Paape, and T. Wanner. Spinodal decomposition for the Cahn-HilliardCook equation. Communications in Mathematical Physics, 223(3):553-582, 2001.

[3] D. Blömker, S. Maier-Paape, and T. Wanner. Phase separation in stochastic Cahn-Hilliard models. Nova Science Publishers, Mathematical Methods and Models in Phase Transitions:1$41,2005$.

[4] D. Blömker, S. Maier-Paape, and T. Wanner. Second phase spinodal decomposition for the Cahn-Hilliard-Cook equation. Trans. Amer. Math. Soc., 360(1):449-489 (electronic), 2008.

[5] J. F. Blowey and C. M. Elliott. The Cahn-Hilliard gradient theory for phase separation with nonsmooth free energy. I. Mathematical analysis. European J. Appl. Math., 2(3):233-280, 1991.

[6] J. W. Cahn. On spinodal decomposition. Acta Metallurgica, 9(9):795-801, September 1961.

[7] J. W. Cahn and J. E. Hilliard. Free energy of a nonuniform system. i. interfacial free energy. Journal of Chemical Physics, 28(2):258, February 1958.

[8] J. W. Cahn and J. E. Hilliard. Spinodal decomposition: a reprise. Acta Metallurgica, 19(2):151-161, February 1971.

[9] C. Cardon-Weber. Cahn-Hilliard stochastic equation: existence of the solution and of its density. Bernoulli, 7(5):777-816, 2001.

[10] C. Cardon-Weber. Cahn-Hilliard stochastic equation: strict positivity of the density. Stoch. Stoch. Rep., 72(3-4):191-227, 2002.

[11] H. Cook. Brownian motion in spinodal decomposition. Acta Metallurgica, 18:297-306, 1970.

[12] G. Da Prato and A. Debussche. Stochastic Cahn-Hilliard equation. Nonlinear Anal., 26(2):241-263, 1996.

[13] G. Da Prato and J. Zabczyk. Stochastic equations in infinite dimensions, volume 44 of Encyclopedia of Mathematics and its Applications. Cambridge University Press, Cambridge, 1992.

[14] R. C. Dalang, C. Mueller, and L. Zambotti. Hitting properties of parabolic s.p.d.e.'s with reflection. Ann. Probab., 34(4):1423-1450, 2006.

[15] A. Debussche and L. Dettori. On the Cahn-Hilliard equation with a logarithmic free energy. Nonlinear Anal., 24(10):1491-1514, 1995.

[16] A. Debussche and L. Goudenège. Stochastic Cahn-Hilliard equation with double singular nonlinearities and two reflections. SIAM J. Math. Anal., 43(3):1473-1494, 2011.

[17] A. Debussche and L. Zambotti. Conservative stochastic Cahn-Hilliard equation with reflection. Ann. Probab., 35(5):1706-1739, 2007.

[18] N. Elezović and A. Mikelić. On the stochastic Cahn-Hilliard equation. Nonlinear Anal., 16(12):1169-1200, 1991.

[19] T. Funaki and S. Olla. Fluctuations for $\nabla \phi$ interface model on a wall. Stochastic Process. Appl., 94(1):1-27, 2001.

[20] G. Giacomin, S. Olla, and H. Spohn. Equilibrium fluctuations for $\nabla \phi$ interface model. Ann. Probab., 29(3):1138-1172, 2001. 
[21] L. Goudenège. Stochastic Cahn-Hilliard equation with singular nonlinearity and reflection. Stochastic Process. Appl., 119(10):3516-3548, 2009.

[22] C. P. Grant. Spinodal decomposition for the Cahn-Hilliard equation. Comm. Partial Differential Equations, 18(3-4):453-490, 1993.

[23] M. Hairer and J. C. Mattingly. Ergodicity of the 2D Navier-Stokes equations with degenerate stochastic forcing. Ann. of Math. (2), 164(3):993-1032, 2006.

[24] M. Hairer and J. C. Mattingly. A theory of hypoellipticity and unique ergodicity for semilinear stochastic PDEs. Electron. J. Probab., 16:no. 23, 658-738, 2011.

[25] J. S. Langer. Theory of spinodal decomposition in alloys. Annals of Physics, 65:53-86, 1971.

[26] S. Maier-Paape and T. Wanner. Spinodal decomposition for the Cahn-Hilliard equation in higher dimensions. I. Probability and wavelength estimate. Comm. Math. Phys., 195(2):435464, 1998.

[27] S. Maier-Paape and T. Wanner. Spinodal decomposition for the Cahn-Hilliard equation in higher dimensions: nonlinear dynamics. Arch. Ration. Mech. Anal., 151(3):187-219, 2000.

[28] A. Novick-Cohen. The Cahn-Hilliard equation: mathematical and modeling perspectives. Adv. Math. Sci. Appl., 8(2):965-985, 1998.

[29] A. Novick-Cohen and L. A. Segel. Nonlinear aspects of the Cahn-Hilliard equation. Phys. D, 10(3):277-298, 1984.

[30] D. Nualart and É. Pardoux. White noise driven quasilinear SPDEs with reflection. Probab. Theory Related Fields, 93(1):77-89, 1992.

[31] Y. Otobe. Stochastic partial differential equations with two reflecting walls. J. Math. Sci. Univ. Tokyo, 13(2):129-144, 2006.

[32] E. Sander and T. Wanner. Monte Carlo simulations for spinodal decomposition. J. Statist. Phys., 95(5-6):925-948, 1999.

[33] E. Sander and T. Wanner. Unexpectedly linear behavior for the Cahn-Hilliard equation. SIAM J. Appl. Math., 60(6):2182-2202 (electronic), 2000.

[34] T. Wanner. Maximum norms of random sums and transient pattern formation. Trans. Amer. Math. Soc., 356(6):2251-2279 (electronic), 2004.

[35] L. Zambotti. Integration by parts formulae on convex sets of paths and applications to SPDEs with reflection. Probab. Theory Related Fields, 123(4):579-600, 2002.

[36] L. Zambotti. Integration by parts on $\delta$-Bessel bridges, $\delta>3$ and related SPDEs. Ann. Probab., 31(1):323-348, 2003.

[37] L. Zambotti. Fluctuations for a $\nabla \phi$ interface model with repulsion from a wall. Probab. Theory Related Fields, 129(3):315-339, 2004.

[38] L. Zambotti. Fluctuations for a conservative interface model on a wall. ALEA Lat. Am. J. Probab. Math. Stat., 4:167-184, 2008. 